This is an informal report intended primarily for internal or limited external distribution. (The opinions and conclusions stated are those of the author and may or may not be those of the laboratory.) This begiven-additional oxternat distribution or eited in external- doeuments without the-eensent of the auther or LLL Technieal Infermation-Department.

UCID - 16431

\section{노 \\ LAWRENCE LIVERMORE LABORATORY \\ University of California/Livermore, California}

AUTO-ACCELERATION IN AN IRIS LOADED PIPE

V. Kelvin Neil and Arthur A. Mirin

December 14, 1973 the United States nor the United States Atomic Energy Commission, nor any of their employees, nor any of their contractors, subcontractors, or their employees, makes any warranty, express or implied, or assumes any legal liability or responsibility for the accuracy, completeness or pleteness or urefulnss of any information, apparatus, product or process disclosed, or represents that its use would not infringe privately owned rights. 


\section{DISCLAIMER}

This report was prepared as an account of work sponsored by an agency of the United States Government. Neither the United States Government nor any agency Thereof, nor any of their employees, makes any warranty, express or implied, or assumes any legal liability or responsibility for the accuracy, completeness, or usefulness of any information, apparatus, product, or process disclosed, or represents that its use would not infringe privately owned rights. Reference herein to any specific commercial product, process, or service by trade name, trademark, manufacturer, or otherwise does not necessarily constitute or imply its endorsement, recommendation, or favoring by the United States Government or any agency thereof. The views and opinions of authors expressed herein do not necessarily state or reflect those of the United States Government or any agency thereof. 


\section{DISCLAIMER}

Portions of this document may be illegible in electronic image products. Images are produced from the best available original document. 


\title{
AITO-ACCELERATION IN AN IRIS LOADED PIPE
}

\author{
V. Kelvin Neil and Arthur A, Mirin
}

December 14,1973 .

The idea of auto-acceleration is not new. ${ }^{1-5}$ The concept entails a transfer of energy from one group of particles to another group. As an example, the forward portion of a burst of relativistic electrons may leave in its wake electromagnetic energy stored in a surrounding structure, thus reducing the particles' kinetic energy. This stored energy is subsequently imparted to particles in the backward portion of the burst. A conceptually ideal structure would be one characterized by an inductance per unit length $L$, so that the electric field $E$ along the beam is given by

$$
E=-\mathrm{Ld} / \mathrm{d} t
$$

where $I$ is the beam current

Unfortuantely, no such ideal structure exists. Any practical

structure will have inductance, capactiance, and resistance, generally all varying with frequency. . Thus the structure will have characteristic electromagnetic modes that are excited by the time-varying current. If the current varies slowly enough, the excitation of these modes will be negligible, and 
Eq. (1) is indeed valid. This slowly varying current constitutes what we shall call the "adiabatic" regime of auto-acceleration.

On the other hand, a current which varies rapidly enough to excite the eigenmodes constitutes what we shall call the "rf" regime of auto-acceleration. If the electric field at the beam arises primarily from the excitation of a single mode, it will vary sinusoidally at the eigenfrequency of the mode. Within a length along the beam equal to one free-space wavelength of the mode, some particles may gain energy while others lose energy. Qualitatively the same situation prevails if several modes of the structure are excited. In the rf regime the energy modulation of beam particles may lead to a modulation of the charge per unit length and of the beam current. The modulated current excites the structure even more, and an instability can result. ${ }^{6}$.The rf regime then must be divided into two parameter regions, with and without growth of the unstable current modulation. On the basis of the linear theory in Ref. 6, the instability will always occur if the structure has an infinite quality factor, $Q$. In this work we have set $Q=\infty$, so that it is not too surprising that the calculation described in Section IV, which treats a current increasing linearly with time, exhibits this instability.

In Section V we discuss the possibility of properly "tailoring" the beam current vs time to achieve favorable coupling to the structure, No longitudinal instability is encountered in the relatively short beam treated. 


\section{The Structure and Electric Field}

In this work we consider the process of auto-acceleration in an iris loaded pipe as shown in Fig. 1. Each section of this structure is treated as a separate cavity with characteristic eigenmodes. The modes contributing to exchange of energy among particles are the azimuthally symmetric TM modes. Only these modes with no axial (z) dependence will be considered. There are, of course, other modes. In particular, the TM modes with one wavelength variation in the azimuthal direction (dipole, or beam break-up modes) can be quite troublesome. ${ }^{6,7}$

For purposes of describing the electromagnetic fields in each cavity, we neglect the presence of the hole, consequently neglecting transfer of energy between cavities by all means except the electron beam. We further neglect the finite resistance in the cavity surfaces, which is a good approximation if the times of interest are much shorter than the filling time of the modes.

Our model of the electron beam has radially uniform current density out to radius $r=a$ and zero beyond. The current density varies with $z$ and $t$. The electric field generated by the beam charge is not considered. This electric field will terminate on the fins near the inner edge, and should have little effect on particles' axial motion if the variation in the charge per unit length $\lambda$ is small over a distance comparable to the radius of the hole.

To calculate the electromagnetic fields generated by the beam current, we represent the normal modes of the cavity by their vector 
potentials $\bar{A}_{\ell}(\bar{r})$. For the pertinent modes we have

$$
\bar{A}_{\ell}(\overline{\hat{r}})=N_{\ell} J_{o}\left(\omega_{\ell} r / a\right) \hat{z}
$$

with $\mathrm{N}_{\ell}$ a normalization constant and $\omega_{\ell}$ the eigenfrequency of the mode. The eigenfrequencies are determined from the condition $J_{0}\left(\omega_{\ell} b / c\right)=0$, where $b$ is the radius of the pipe wall. The normalization condition is standard, ${ }^{4}$ namely

$$
\int \bar{A}_{\ell} \cdot \bar{A}_{\ell}, d v=4 \pi c^{2} \delta_{l \ell !},
$$

with the integral extending over the volume of the cavity. Guassian units are used in this part of our calculation. From Eqs. (2) and (3) we obtain

$$
N_{\ell}^{2}=4 c^{2} / h b^{2} J_{1}^{2}\left(x_{\ell}\right)
$$

in which $x_{\ell}$ is the $\ell$ th root of $J_{Q}$, and $h$ is the axial separation of the fins. The actual vector potential in the cavity, $A(r, t)$, may be expressed as a sum over the eigenmodes. We have

$$
A(r, t)=\sum_{\ell} q_{\ell}(t) N_{\ell} J_{0}\left(x_{\ell} r / b\right) .
$$

. It is shown in Ref. 6 and elsewhere that, for perfectly conducting walls, the time dependent coefficients $q_{\ell}$ obey the equation 


$$
\ddot{q}_{\ell}+\omega_{\ell}^{2} q_{\ell}=\frac{1}{c} \int \bar{j}(r, t) \cdot \bar{A}_{\ell}(\bar{r}) d v .
$$

We take the beam current to be

$$
\bar{j}(r, t)=\frac{I(t)}{\pi a^{2}} \Theta(a-r)
$$

and with the use of Eq. (2) in Eq. (6) we obtain

$$
\ddot{q}_{\ell}+\omega_{\ell}^{2} q_{\ell}=\frac{2 h}{c} \frac{b}{a} \frac{N_{l}}{x_{\ell}} J_{1}\left(x_{\ell} a / b\right) I(t) .
$$

Equation (8) is to be solved with the initial conditions $q_{l}=0, \dot{q}_{l}=0$ at $t=0$. The solution is

$$
q_{\ell}(t)=\frac{2 h b N_{\ell} J_{1}\left(x_{\ell} a / b\right)}{\operatorname{cax}_{\ell}(1)} \int_{0}^{t} I\left(t^{\prime}\right) \sin \omega_{\ell}\left(t-t^{\prime}\right) d t^{\prime} .
$$

The electric field from the $\mathrm{lth}$ mode is given by

$$
\bar{E}_{\ell}(r, t)=-\frac{\dot{q}_{\ell}}{c} N_{\ell} J_{o}\left(x_{\ell} r / b\right) \hat{z} .
$$

We evaluate this field at the beam radius, $r=a$, to obtain (inserting also the value of $\mathrm{N}_{\ell}^{2}$ from Eq. (4))

$$
E_{\ell}(r=a, t)=-\frac{8 J_{0}\left(x_{\ell} a / b\right) J_{1}\left(x_{\ell} a / b\right)}{a b x_{\ell} J_{1}^{2}\left(x_{\ell}\right)} \int_{0}^{t} I\left(t^{\prime}\right) \cos \omega_{\ell}\left(t-t^{\prime}\right) d t^{\prime} .
$$


For a given $I(t)$ the electric field can be determined. For a current increasing linearly with time, we have $I(t)=I t / T$, and the integral in Eq. (11) is easily performed with the result

$$
\int_{0}^{t} I\left(t^{\prime}\right) \cos \omega_{\ell}\left(t-t^{\prime}\right) d t^{\prime}=\frac{I}{\omega_{\ell} T}\left(1-\cos \omega_{\ell} t\right) .
$$

The electric field calculated in this manner has been evaluated computationally, with the sum extending over the first 50 modes. Results are shown in practical units in Fig. 2 for $a=1$ inch, $b=6$ inches, and $\mathrm{T}=10 \mathrm{~ns}$. For $\mathrm{I}$ of $17 \mathrm{kA}$ the peaks of the electric field are about $11 \mathrm{kV} / \mathrm{cm}$. From Eqs. (11) and (12) we may calculate the electric field $E_{1}$ from the first mode only. We find $E_{1}(t)=-4\left(1-\cos \omega_{\ell} t\right)$ in $\mathrm{kV} / \mathrm{cm}$. It is interesting to compare this result with the "ideal" structure mentioned above. We may take the inductance per unit length $\mathrm{L}$ to be given by (practical units, now)

$$
L=\frac{\mu_{0}}{2 \pi} \cdot \ln (b / a) .
$$

For $\mathrm{b} / \mathrm{a}=6$, we have $\mathrm{L}=3.6 \times 10^{-7}$ henries/m. Fmploying this value and $\mathrm{a}$ current of $17 \mathrm{kA}$ rising in $10 \mathrm{~ns}$ in Eq. (1) we find $\mathrm{E}=-6.1 \mathrm{kV} / \mathrm{cm}$. This value is approximately the average of the results shown in Fig. 2. An important characteristic of the electric field is its periodic structure, which can lead to a longitudinal instability discussed above. 


\section{Particle Dynamics}

We consider only the axial motion of the electrons. This simplification is in part justified if we assume a large axial magnetic field, which inhibits transverse motion of the particles. . It is convenient to employ the hydrodynamic equations in their Lorentz invariant form. We introduce the quantities $u$ and $\mu$ by the definitions

$$
\begin{aligned}
& u=r v / c, \\
& \mu=r_{0} n / \gamma,
\end{aligned}
$$

in which the symbols mean:

$$
\begin{aligned}
\gamma & =\text { particles' energy in units of rest energy } \\
v & =\text { particles' speed } \\
\mathrm{c} & =\text { speed of light } \\
\mathrm{n} & =\text { number of particles per unit length in beam } \\
\mathrm{r}_{\mathrm{o}} & =\text { particles' classical radius. }
\end{aligned}
$$

In terms of the above quantities, the beam current $I$ is given by

$$
I=e c \mu u / r_{0}
$$

with e the particles' charge, taken to be positive. The quantities $u$ and $\mu$ are functions of $z$ and $t$, and $\gamma$ is related to $u$ by the equation. 


$$
\gamma^{2}=1+u^{2}
$$

The Eulerian equation of motion can be expressed as

$$
\left(\frac{\gamma}{c} \cdot \frac{\partial}{\partial t}+u \frac{\partial}{\partial z}\right) \gamma=e u E / m c^{2},
$$

or

$$
\left(\frac{\dot{y}}{c} \frac{\partial}{\partial t}+u \frac{\partial}{\partial z}\right) u=e \gamma E / m c^{2} \text {, }
$$

in which $m$ is the particles' rest mass. The electric field $F$ is a sum over the electric fields of the normal modes excited by the beam. We have at any position $z_{i}$,

$$
E\left(z_{i}, t\right)=\sum_{\ell} E_{\ell}\left(z_{i} t\right),
$$

with $E\left(z_{i}, t\right)$ the field in a cavity located at $z_{i}$. Equation (11) gives $E_{\ell}\left(z_{i}, t\right)$. The form of Eq. (11) suggests using a dimensionless time $\tau$ and position $y$ given by

$$
\begin{aligned}
\tau & =c t / b, \\
y & =z / b .
\end{aligned}
$$

Inserting these definitions as well as Eq. (15) into Eq. (11), we have 


$$
E_{l}=-\frac{8 J_{1}\left(x_{l} a / b\right) J_{0}\left(x_{l} a / b\right) e}{r_{0} x_{l} a J_{1}^{2}\left(x_{l}\right)} \int_{0}^{\tau} \mu u \cos x_{l}\left(\tau-\tau^{\prime}\right) d \tau^{\prime} .
$$

We multiply both sides of Eq. (18) by b and insert Eqs. (19) and (22) to obtain

$$
\begin{aligned}
\left(\frac{\gamma \partial}{\partial \tau}+u \frac{\partial}{\partial y}\right) u= & -8 \gamma \sum_{\ell} \frac{J_{1}\left(x_{\ell} a / b\right) J_{0}\left(x_{\ell} a / b\right)}{\left(x_{\ell} a / b\right) J_{1}^{2}\left(x_{\ell}\right)} \\
& x \int_{0}^{\tau} \mu u \cos x_{\ell}\left(\tau-\tau^{\prime}\right) d \tau^{\prime}
\end{aligned}
$$

The continuity equation may be written in the form

$$
\frac{1}{c} \frac{\partial}{\partial t}(\mu \gamma)+\frac{\partial}{\partial z}(\mu u)=0
$$

In terms of $\tau$ and $y$, we have

$$
\frac{\partial}{\partial \tau}(\mu \gamma)+\frac{\partial}{\partial y}(\mu u)=0
$$

Equations (24) and (25) are solved numerically, with $u(0, t)$ and $\mu(0, t)$ specified for all time. The dimensions used are $b=6$ in, $a=1$ in. The grid spacing in $y$ is $1 / 6$, corresponding to 1 in spacing in $z$. The total length of the $z$ grid is 96 in. The differencing of these equations is described in the next section. 
III. Differencing of Equations

We let

$$
\lambda=\mu \gamma
$$

and

$$
\tilde{E}=\frac{r_{0} b}{e} \sum_{\ell} E_{\ell} .
$$

Equations (23) and (25) may be rewritten:

$$
\begin{aligned}
& \frac{\partial}{\partial \tau}(\lambda)+\frac{\partial}{\partial y}(\mu u)=0 \\
& \frac{\partial}{\partial \tau}(u)+\frac{u}{\gamma} \frac{\partial}{\partial y}(u)=\tilde{E} .
\end{aligned}
$$

Denote the $y$ grid spacing by $\Delta y$, and the normalized time step by $\Delta \tau$. Let

$$
y_{j}=(j-1) \Delta y \quad j=1, \ldots, J
$$

and

$$
\tau^{n}=n \Delta \tau \quad n \geq 0 .
$$


Abbreviate

$$
f_{j}^{n}=f\left(y_{j}, \tau^{n}\right)
$$

for any dependent variable $f(y, \tau)$.

A two-step Lax-Wendroff method is used at the interior mesh points.

The first step is:

$$
\begin{aligned}
\lambda_{j+1 / 2}^{n+1 / 2}= & \frac{1}{2}\left(\lambda_{j}^{n}+\lambda_{j+1}^{n}\right)-\frac{\Delta \tau}{2 \Delta y}\left((\mu u)_{j+1}^{n}-(\mu u)_{j}^{n}\right) \\
u_{j+1 / 2}^{n+1 / 2}= & \frac{1}{2}\left(u_{j}^{n}+u_{j+1}^{n}\right) \\
& -\frac{\Delta \tau}{2 \Delta y}\left(\frac{u_{j}^{n}+u_{j+1}^{n}}{\gamma_{j}^{n}+\gamma_{j+1}^{n}}\right)\left(u_{j+1}^{n}-u_{j}^{n}\right) \\
& +\frac{\Delta \tau}{4}\left(\tilde{E}_{j}^{n}+\tilde{E}_{j+1}^{n}\right) . \quad j=1, \ldots, J-1 .
\end{aligned}
$$

The second step is

$$
\begin{aligned}
\lambda_{j}^{n+1}= & \lambda_{j}^{n}-\frac{\Delta \tau}{\Delta y}\left((\mu u)_{j+1 / 2}^{n+1 / 2}-(j u)_{j-1 / 2}^{n+1 / 2}\right) \\
u_{j}^{n+1}= & u_{j}^{n}-\frac{\Delta \tau}{\Delta y}\left(\frac{u_{j-1 / 2}^{n+1 / 2}+u_{j+1 / 2}^{n+1 / 2}}{\gamma_{j-1 / 2}^{n+1 / 2}+\gamma_{j+1 / 2}^{n+1 / 2}}\right)\left(u_{j+1 / 2}^{n+1 / 2}-u_{j-1 / 2}^{n+1 / 2}\right) \\
& +\frac{\Delta \tau}{2}\left(\tilde{E}_{j-1 / 2}^{n+1 / 2}+\tilde{E}_{j+1 / 2}^{n+1 / 2}\right) \cdot \quad J=2, \ldots, J-1 .
\end{aligned}
$$


The right hand boundary point $\mathrm{j}=\mathrm{J}$ is computed via the one sided scheme

$$
\begin{gathered}
\lambda_{J}^{n+1}=\lambda_{J}^{n}-\frac{\Delta \tau}{\Delta y}\left((\mu u)_{J}^{n}-(\mu u)_{J-1}^{n}\right) \\
u_{J}^{n+1}=u_{J}^{n}-\frac{\Delta \tau}{\Delta y} \frac{u_{J}^{n}}{\gamma_{J}^{n}}\left(u_{J}^{n}-u_{J-1}^{n}\right) \\
+\Delta \tau \tilde{E}_{J}^{n} .
\end{gathered}
$$

The values of $\lambda$ and $\mu$ at the left hand boundary point $j=1$ are specified at all times.

The stability criterion is

$$
\frac{\Delta \tau}{\Delta y} \leq \min _{j} \frac{\gamma_{j}}{u_{j}} .
$$

We let

$$
\Delta \tau=\frac{1}{2} \Delta y
$$


IV. Results for Linearly Rising Current

We treat a beam current rising linearly with time at the rate of $1700 \mathrm{~A} / \mathrm{ns}$. The initial energy of the particles is such that $\gamma=6$. Results 2 ns after the start of the calculation are shown in Fig. 3. The top graph is $\mu \gamma$ vs $z$, the middle graph is $\gamma$ vs $z$, and the bottom graph is $E_{1}$ vs $z$, where $E_{1}$ is the electric field from the first mode only (i.e., the first term in the sumnation in Eq. (19)), Recall that $\mu \gamma$ is a measure of the linear charge density in the beam, and that $\mu \gamma=1$ corresponds to $17,000 \mathrm{~B}$ amperes. The value of $\gamma$ is calculated for the entire length. At this early stage of the calculation, the behavior of $E_{1}$ is very nearly that calculated from a given current, neglecting the action of the field on the particles.

This calculation was continued, and results at 9 ns after the start of injection are shown in Fig. 4. These results give some insight into the non-linear behavior of the longitudinal bunching. The instability discussed above has certainly played a role in the calculation. The largest values of $E_{1}$ and $\gamma$ are found around halfway between the injection point and the head of the beam, a behavior predicted by linear theory. The peak values of the electric field are enhanced by about a factor of 4 over those at $2 \mathrm{~ns}$, and there are regions of strong accelerating electric fields. On the average, particles have lost energy, but a sizable fraction have gained energy.

It is of interest to compare these results with those expected if the structure had behaved "adiabatically", that is, with an electric field given by Eq. (1) with $L=3.6 \times 10^{-7}$ henries $/ \mathrm{m}$. The electric field 
would be uniform with a value of about $-6 \mathrm{kV} / \mathrm{cm}$. Particles near the head of the beam would have experienced a decelerating field of $\sim 15 \mathrm{kV} / \mathrm{in}$ for about 90 inches and lost $1.4 \mathrm{MeV}$ of kinetic energy. The plot of $\gamma$ would be monotonically decreasing from 6 at $z=0$ to about 3 at $z=90$ in.

The results shown in Fig. 4 suggest that large accelerating electric fields may be obtained by allowing unstable axial motion to occur. But the trouble. with allowing instabilities to take over is that the usual result is chaos.

of one puts faith in the linear theory, it seems possible to control growth of the instability by chosing the value of $Q$ for the structure.

The results shown in Fig. 5 are for the same calculation as in Fig. 4, except that the first 20 modes of the cavities are used in calculating the electric field. These results show definite peaks in the linear charge density. These peaks are so narrow that the validity of the calculation is questionable because the electrostatic field has been left out. The axial component of this field would tend to suppress the formation of these narrow peaks.

\section{Shaping the Current}

It is theoretically possible to choose a current $I(t)$ such that the first mode of the cavities transfers energy from the front of the beam to the back, and furthermore, no energy is left in the first mode after the beam passes. Some energy will be left in the higher modes. 
The condition that no energy be left in the first mode is satisfied if both $q_{1}$ and $\dot{q}_{1}$ are zero as the tail of the beam leaves the cavity $\left(t=t_{1}\right)$. From Eqs. (9) and (11) we see that the necessary conditions are

$$
\int_{0}^{t_{1}} I(t) \sin \omega_{1}\left(t_{1}-t\right) d t=0
$$

and

$$
\int_{0}^{t_{1}} I(t) \cos \omega_{1}\left(t_{1}-t\right) d t=0 .
$$

If we choose $t_{1}$ such that $\omega t_{1}=3 \pi$, and make $I(t)$ symetric about $t=t_{1} / 2$, then $\sin \omega t_{1}=0$ and a qualitative sketch shows that

$$
\int_{0}^{t_{1}} I(t) \cos \omega_{1} t d t=0 .
$$

If we then further restrict $I(t)$ to be such that

$$
\int_{0}^{t_{1}} I(t) \sin \omega_{1} t d t=0,
$$

we have satisfied both Eqs. (41) and (42).

One example of an $I(t)$ that satisfies the above conditions is $I(t) \propto \sin \left(\omega_{1} t / 3\right)$. A second is a trapezoidal current distribution shown in Fig. (6a). The electric field of the first mode generated by this $I(t)$ is shown in Fig. (6b). The field rises as $1-\cos \omega_{1} t$ for $0<t<t_{1} / 3$, 
varies as $2 \cos \omega_{1}\left[t-\left(t_{1} / 3\right)\right]$ for $t_{1} / 3<t<2 t_{1} / 3$, and is odd about $t=t_{1} / 2$. The maximm electric field is a function only of the slope of the current rise, and not of the magnitude of the current.

This trapezoidal current pulse was used as an initial condition for a numerical calculation. The results for the first mode only are shown in Fig. 7 at 8 ns after start of injection. Initially the calculation showed no energy left in the beam's wake, but the current pulse is altered by the electric field and the pulse, no longer of the proper shape, leaves some energy behind. The acceleration and deceleration of particles proceeds smoothly without the growing longitudinal density fluctuations of the previous calculation.

Results obtained using the first 20 modes are shown in Fig. (8). Although more energy is left behind, qualitatively the results are much the same as those obtained using the first mode only.

For practical purposes, this particular current vs time is not very exciting. One disadvantage is that the absolute upper bound on the achievable energy is just twice the initial energy. By the time the accelerated particles have reached this maximu, the decelerated particles have lost all their energy and the game is over.

The ultimate energy limit can be made as high as desired, however, by choosing the following pulse shape: The current rises linearly to a value $I_{\max }$ in a time $T$ equal to $n$ periods of, the fundamental mode, then falls linearly back to zero in one period. It can be verified that current shape leaves no energy in the first mode. The electric field of the first mode is of the form 


$$
E_{1}=\left\{\begin{array}{l}
-E_{0}\left(1-\cos \omega_{1} t\right), \text { during rise } \\
n E_{0}\left[1-\cos \omega_{1}(t-T)\right], \text { during fall }
\end{array}\right.
$$

with $E_{0}$ a constant determined by the rate of rise of the current. Thus the accelerating field is $\mathrm{n}$ times the decelerating field, and ideally a maximum energy of $\mathrm{n}$ times the initial energy may be achieved.

In principal, the disadvantage to this current shape is that the accelerated particles have an energy spread extending from the maximum energy down to the initial energy. In practice, the disadvantage is that the current will not maintain its proper shape as the decelerated particles lose energy. It may also be difficult to suppress the longitudinal instability if the current rises in several cycles of the fundamental mode. 


\section{REFERENCES}

1. L. N. Kazanskii, A. V. Kisletsov, and A. N. Lebedev, Atomnaya Energiya 30,27 (1971).

2. A. A. Kolomensky, private commuication, 1970 .

3. I. A. Grishaev and A. M. Shenderovich, Sov. Phys.-Tech. Phys. 17, 1871 (1973).

4. M. Friedman, Phys. Rev. Letters 31, 1107 (1973).

5. N. R. Heese and W. R. Raudorf, Rev. Sci. Instr. 43, 1594 (1972).

6. V. K. Neil and R. K. Cooper, Particle Acc. 1, 111 (1970).

7. W. K. H. Panofsky and M. Bander, Rev. Sci. Instr. 39, 206 (1968). 


\section{FIGURE CAPTIONS}

Fig. 1: Schematic of structure.

Fig. 2: The electric field generated in the first 50 cavity modes by a current rising linearly at the rate of $1700 \mathrm{~A} / \mathrm{ns}$. The observation radius is the beam's outer radius ( 1 in). The abscissa is $\omega_{1} t$, where $\omega_{1}$ is the frequency of the first mode of the cavity. $\left(\omega_{1} t=2 \pi\right.$ corresponds to $\left.t=1.3 \mathrm{~ns}.\right)$

Fig. 3: The quantities $\mu \gamma, \gamma$, and $E_{1}$ vs $z$ at 2 ns after start of injection. The injected current rises linearly with time at the rate of $1700 \mathrm{~A} / \mathrm{ns}$.

Fig. 4: Same calculation as in Fig. 3, but at 9 ns after start of injection.

Fig. 5: Same calculation as in Fig. 4, but the first 20 modes are employed in calculating the electric field $\mathrm{E}$.

Fig. 6a: Current vs time that leaves no energy in the first cavity mode.

Fig, fh: Flertric field (of the first mode) vs time generated by the current in (a). The outer wall is at radius 6 in, and the observation point is at the beam radius ( 1 in).

Fig. 7: The quantities $\mu \gamma, \gamma$, and $E_{1}$ vs $z$ at 8 ns after start of injection. The injected current pulse is shown in Fig. 6a. Recall $\mu \gamma=0.1$ corresponds to $1700 \beta$ amperes.

Fig. 8: Same calculation displayed in Fig, 7, except that the first 20 modes are employed. 


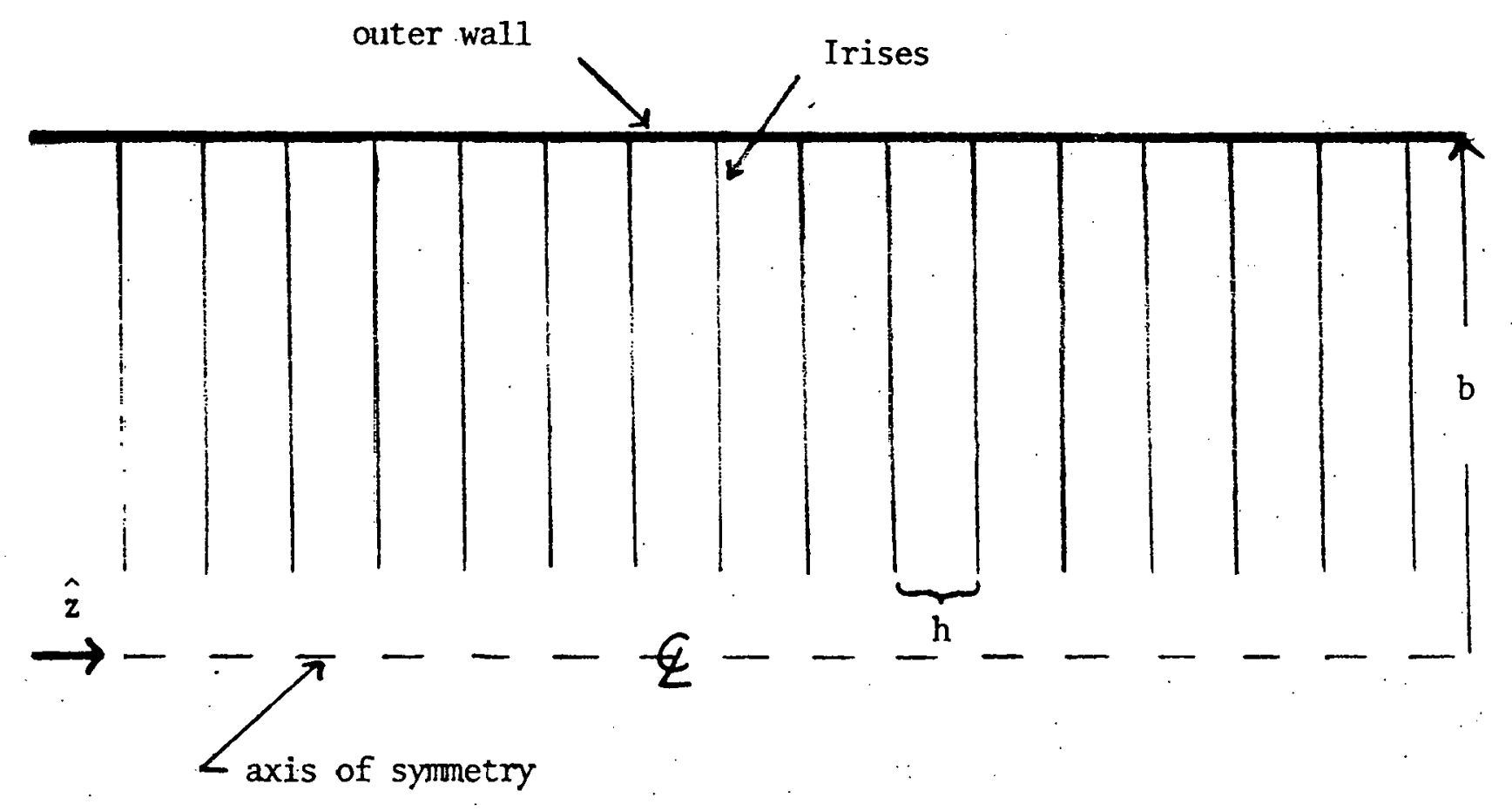

Figure 1 


$$
\text { ann }
$$



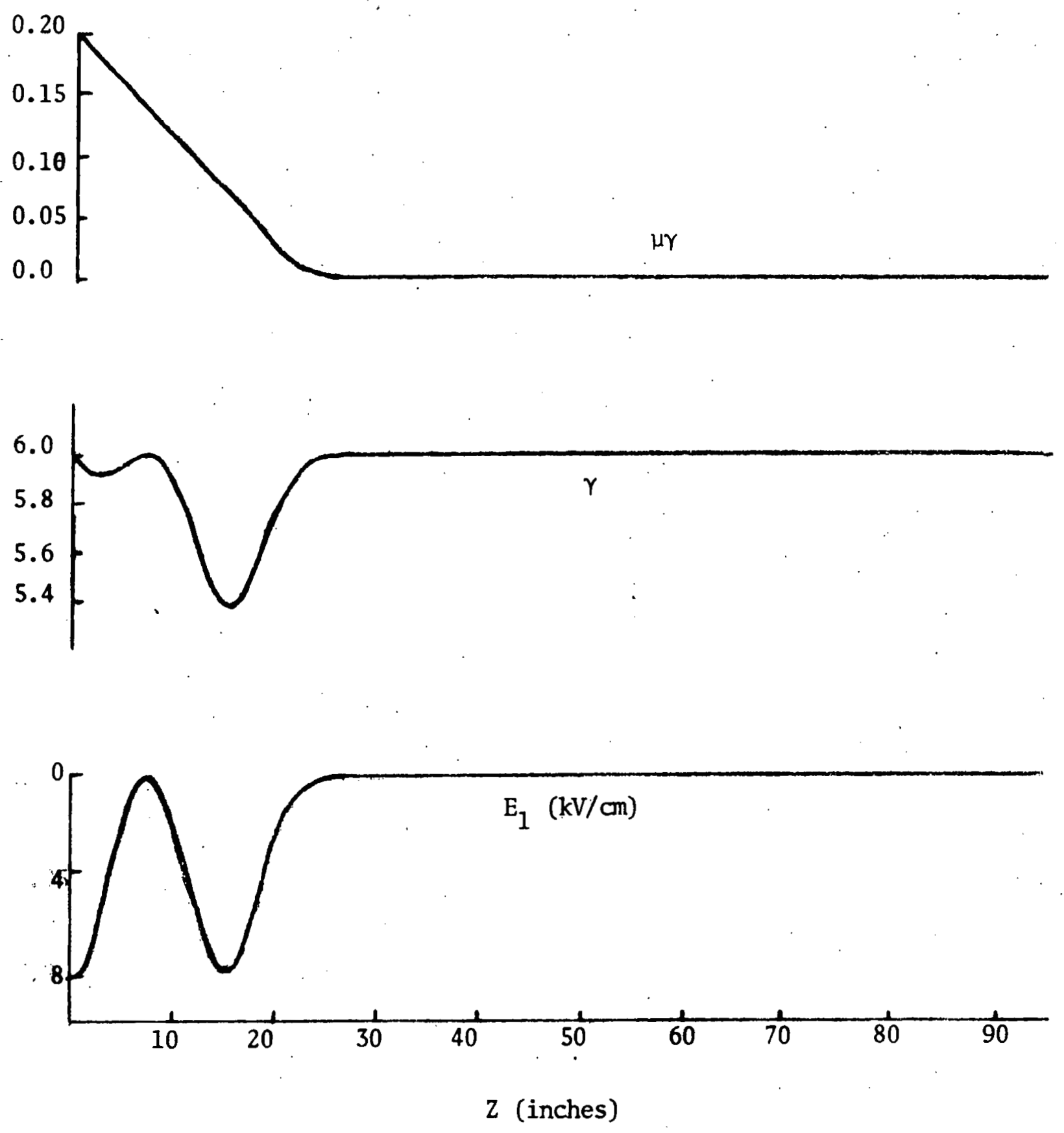

Figure 3 

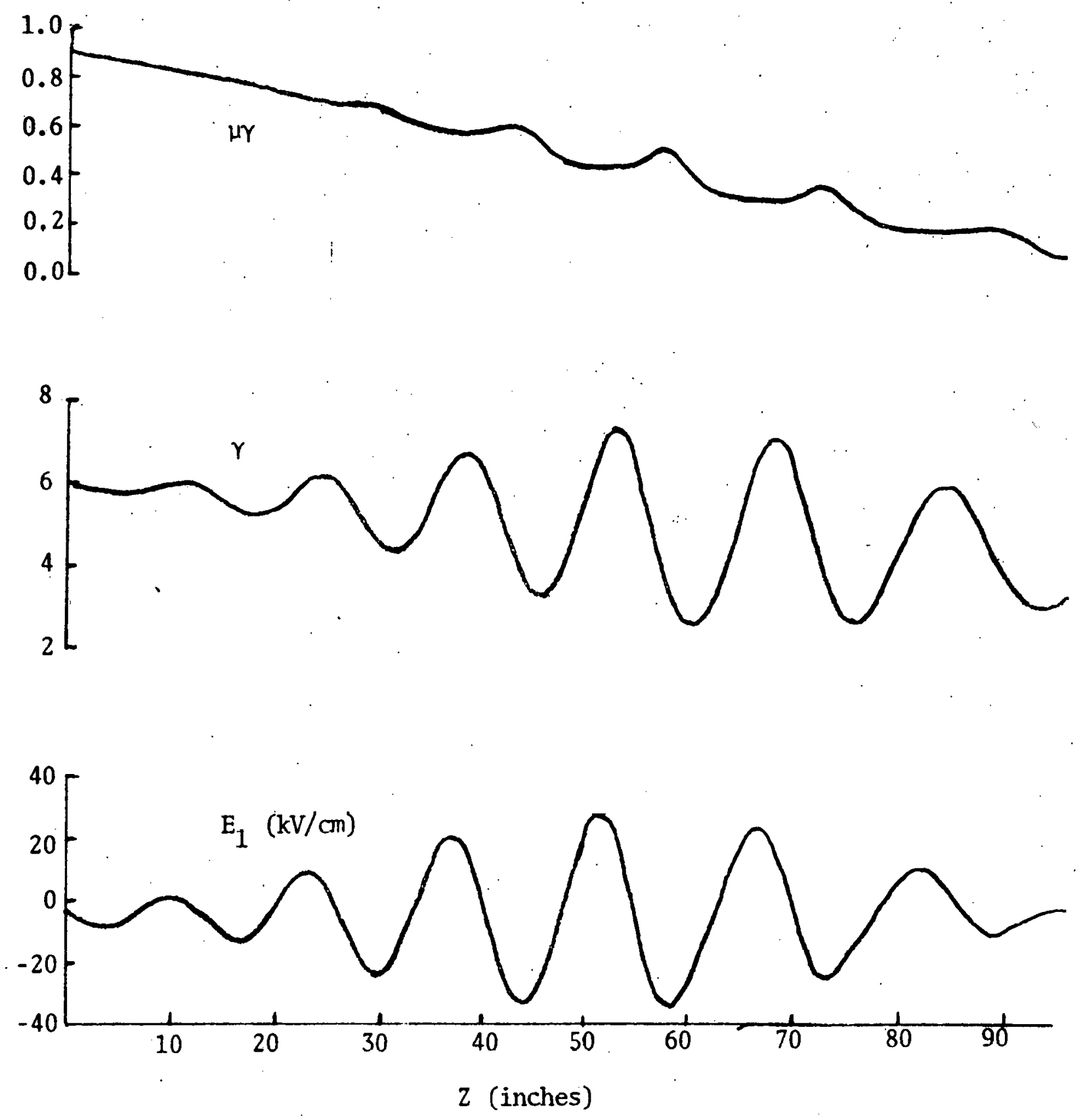

Figure 4 

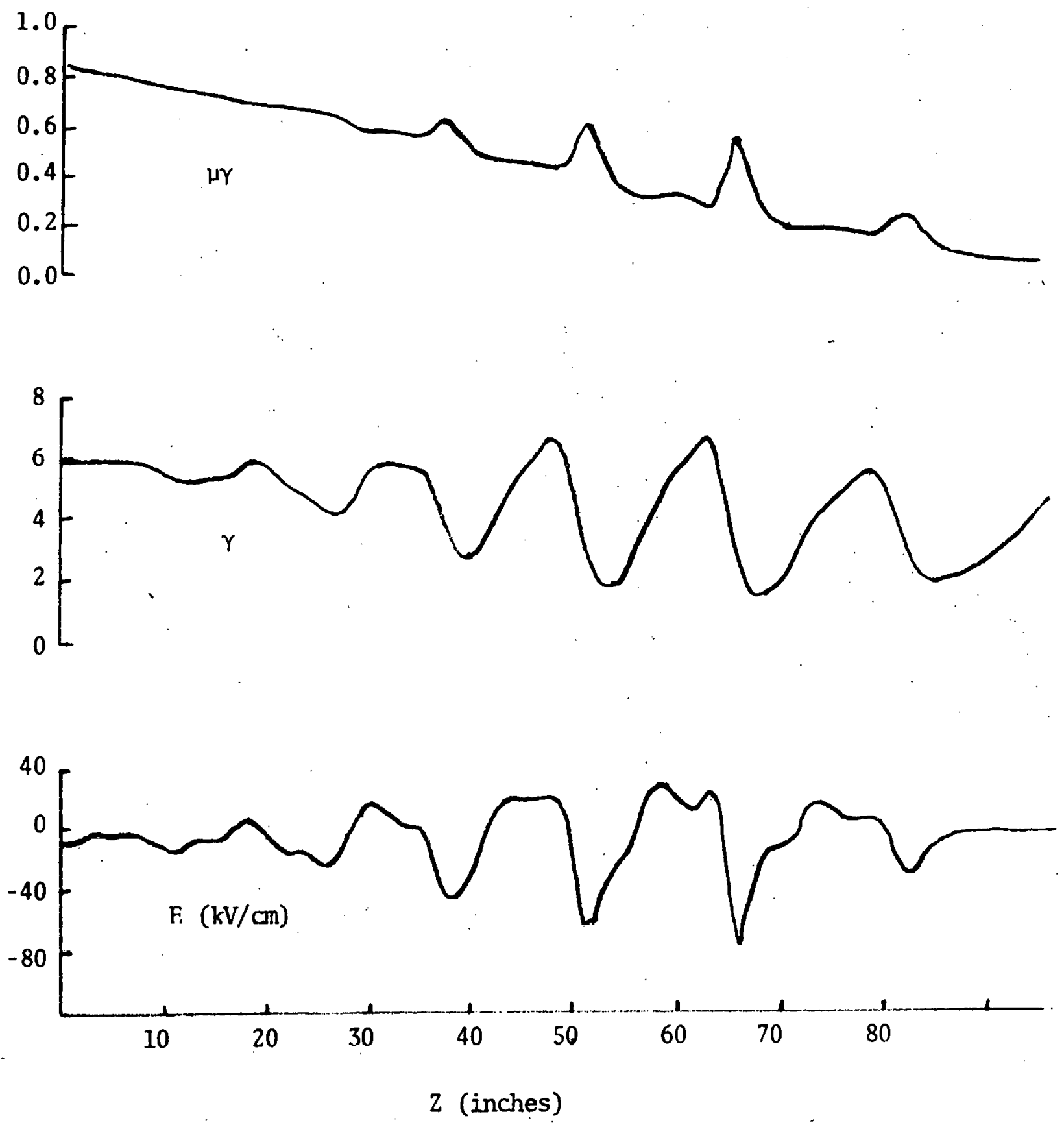

Figure 5 

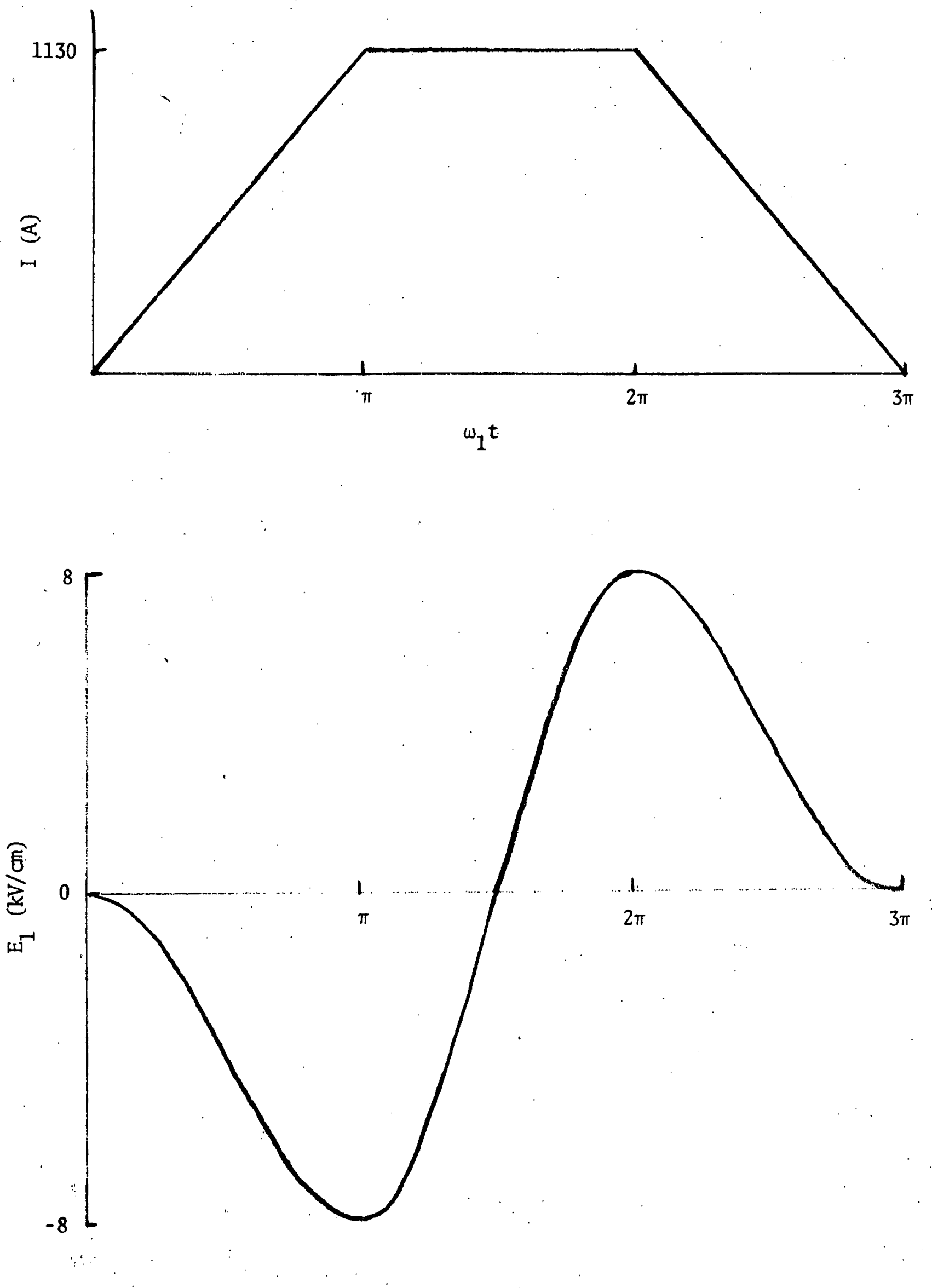

$\therefore \quad \because$

Figure 6 

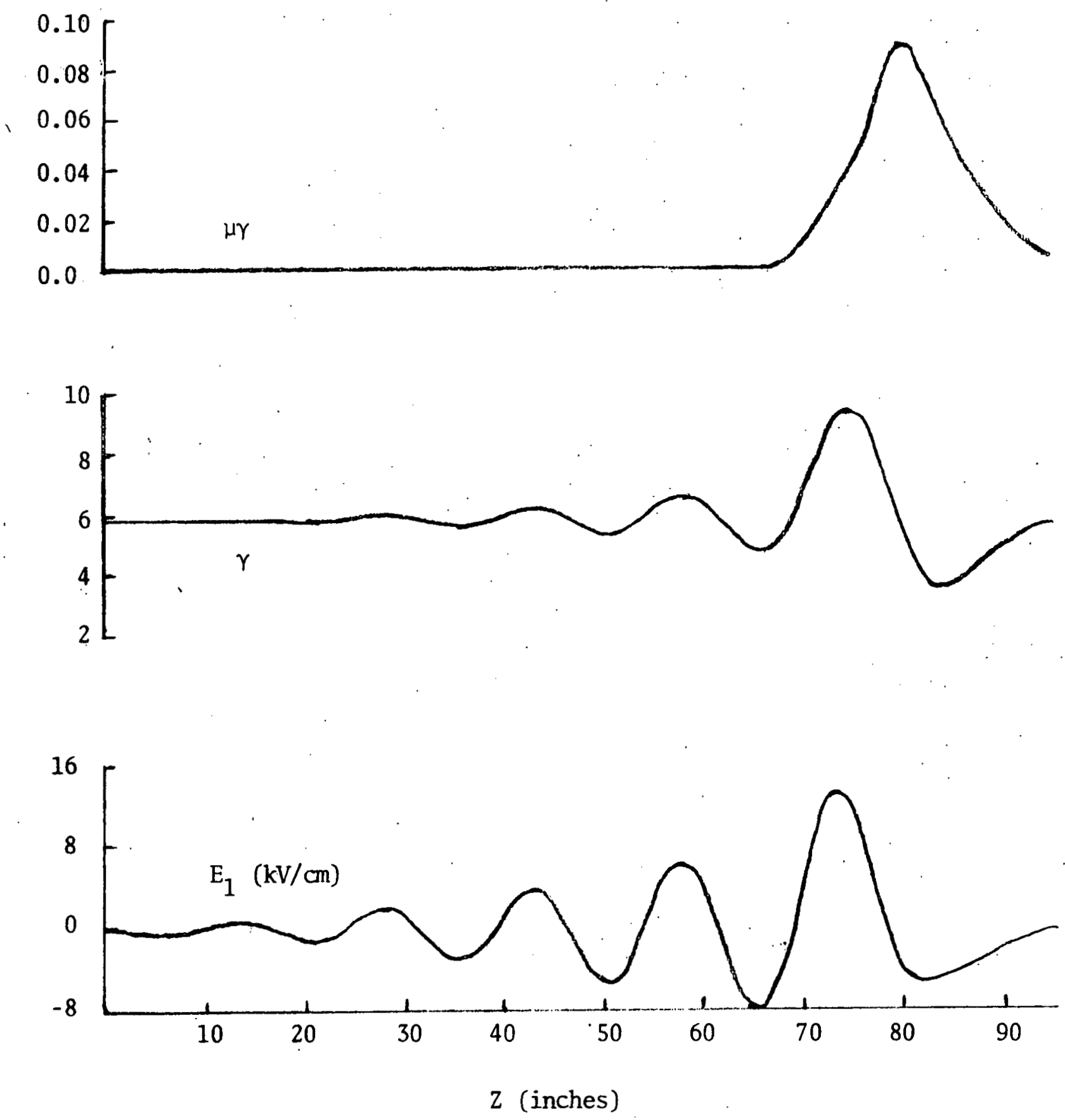

Figure 7 

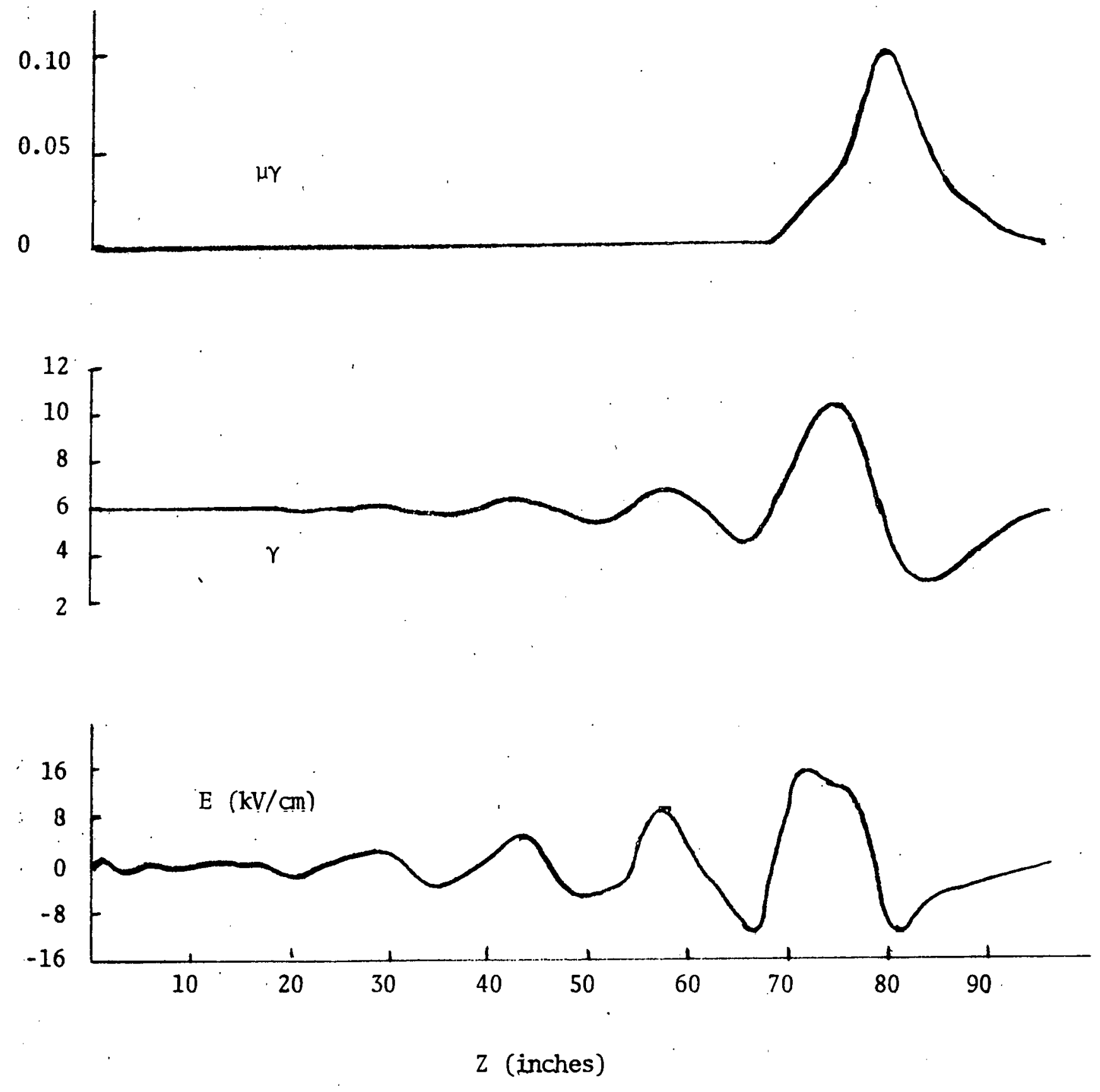

Figure 8 


\section{LLL Interna1}
J. W. Beal
$\mathrm{L}-382$
R. J. Briggs L-382
R. R. Buntzen , $\mathrm{L}-212$
J. C. Clark L-153
T. J. Fessenden L-382
T. K. Fowler L-382
R. E. Hester L-382
J. Killeen $\mathrm{L}-388$
W. A. Lamb L-306
E. J. Lauer L-382
J. M. Leary L L-388
E. P. Lee L-388
A. A. Mirin L-382
V. K. Neil (15) L-382
L. D. Pearlstein L-388
B. H. Smith : L-728
R. L. Spoerlein L-711
T.I.D. (15) L-9

LBL

J. M. Peterson, B. 47, R. 104

A. M. Sessler, B. 50A, R. 4133

L. Smith, B. 50A, R. 3115

\section{External}

M. S. Friedman

Nava1 Research Laboratory

Washington, D. C. 20375

Dr. Larry Cooper

Office of Naval Research

800 North Quincy Street

Arlington, Virginia 22217

NOTICI:

"This report was prepared as an account of work sponsored hy The United States Government. Neither the United States nor the Unied Sucter

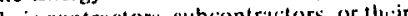
employees, nor any of their contractors employees, makes any warranty. express or implice, or assumes any legal liability or responsibility lor the accuracy, completeness ur useluluess of any intormation, apparatus, product or process disclosed or represents that its use woukd not inlinge privalelyowned rights." 